\title{
Recomendações de Materiais Didáticos Assistida por Computador em Ambientes Virtuais de Aprendizagem
}

\section{Recommendations of Educational Materials for Computer Assisted Learning in Virtual Environments}

\begin{abstract}
Resumo: O artigo objetiva contribuir com discussões de um modelo de sistema de recomendações de materiais didáticos, para ser utilizado em ambientes virtuais de aprendizagem. Também objetiva um resgate teórico de forma a elucidar o modelo apropriado ao protótipo em questão. Foi construído um protótipo de sistema de recomendação para testar o modelo adotado, por meio de um curso de extensão realizado na Faculdade Estácio de Alagoas, no ano de 2010, o qual efetuava gerações baseadas no perfil dos usuários, sendo estas recomendações alvo de análise do estudo. Diante dos estudos efetivados, o modelo de recomendação híbrido proposto apresentou-se satisfatoriamente nos testes iniciais, sendo necessária, como trabalho futuro, a realização novos experimentos para evolução do sistema de recomendação e, consequentemente, geração de recomendações com maior utilidade para o usuário.

Palavras-chave: Educação. Sistemas de recomendações. Protótipo. Ambientes Virtuais de Aprendizagem. Ensino a Distância.
\end{abstract}

\begin{abstract}
The article aims to contribute to the discussion of teaching materials recommendation prototype system' architecture to be used at virtual learning systems. The study also aims to present a literature review that elucidates a method of recommendation suitable for this prototype development. The prototype was built to validate the suggested model, generating recommendation based on student's profile using hybrid model. The study was conducted through an extension course held at the Faculdade Estácio de Alagoas, in 2010. The model has satisfactory results at preliminary tests in this experiment. As future work, new experiments should be done to apprimorate the recommender system in order to produce better results.

Keywords: Education. Recommender systems. Prototype. Virtual Learning Environments. Distance Learning.
\end{abstract}

FERRO, Márcio Robério da Costa; COSTA, Fábio Paraguaçu Duarte da; PERES, Angela Lima; MARINHO, Marcela Ferreira. Recomendações de materiais didáticos assistida por computador em ambientes virtuais de aprendizagem. Informática na Educação: teoria e prática, Porto Alegre, v. 17, n. 1, p. 169-182, jan./jun. 2014.

\author{
Márcio Robério da Costa Ferro \\ Instituto Federal de Educação, Ciências e Tecnologia de Alagoas
}

Fábio Paraguaçu Duarte da Costa Universidade Federal de Alagoas

\section{Angela Lima Peres}

Fundação Universitária de Ciências da Saúde de Alagoas

\section{Introdução}

A evolução da internet proporcionou ao mundo a possibilidade grandes oportunidades, seja por meio de inovadoras modalidades de ensino, como o ensino a distância (EAD), seja pelo desenvolvimento de mercados econômicos interligados, sociedades cada vez mais promissoras.

Cientes dessas influências na vida dos indivíduos, e não diferente no que diz respeito ao ensinar e ao aprender, é que se percebem 
profundas transformações nas relações educacionais, políticas, econômicas, sociais, entre outras. Desse modo, compreende-se que a Educação a Distância (EAD) tem crescido, qualitativa e quantitativamente, nos últimos anos, como explicitado no último Censo da Associação Brasileira de Ensino à Distância (ABED, 2010) e, ainda, compreende-se como explicitado em portaria do Ministério da Educação, No 4059 de 10 de dezembro de 2010, no qual resolve que "[...] as instituições de ensino superior poderão introduzir, na organização pedagógica e curricular de seus cursos superiores reconhecidos, a oferta de disciplinas integrantes do currículo que utilizem modalidade semi-presencial" (BRASIL, 2004).

Maia (2010) caracteriza a modalidade à distância como um processo de desenvolvimento pessoal e profissional, no qual professores e estudantes podem interagir virtualmente por meio da utilização didática das tecnologias da informação e da comunicação. Colaborando com a autora, Almeida (2013) explica os Ambientes Virtuais de Aprendizagem (AVA), no qual a comunicação entre alunos e professores geralmente é realizada, como sendo ambientes computacionais que permitem integrar diversas mídias, permitindo que alunos se comuniquem com seus docentes e tutores, além de permitir o compartilhamento de materiais didáticos das disciplinas, em formato digital. Assim, existe a necessidade de se desenvolve ruma espécie de combinação entre os principais meios de comunicação, objetivando a maior interatividade do aluno com seus professores (FIGUEIREDO; ALMEIDA, 2010).

Relevante destacar que, com o passar do tempo, a quantidade excessiva de material didático disponível pode dificultar a interação entre aluno e ambiente virtual, pois, de acordo com Cazella e Reategui (2006), a grande quan- tidade de informação em meio digital às vezes atrapalha as pessoas que estão à procura de conhecimentos sobre um determinado tema, e essa dificuldade se agrava quando o usuário apresenta pouca ou nenhuma experiência sobre o que está sendo pesquisado. Diante desse cenário parece relevante ressaltar, que há necessidade de criação de ferramentas computacionais de personalização capazes de fornecer aos alunos recomendação de materiais didáticos compatíveis com seus interesses. Assim sendo, sinalizamos os Sistemas de Recomendação, os quais podem possibilitar reduzir a sobrecarga de informações que um usuário recebe ao procurar algum produto, por intermédio da recomendação personalizada, com base no perfil do usuário (ADOMAVICIUS; TUZHILIN, 2005).

Este trabalho trata especificamente da discussão e do modelo de arquitetura, do protótipo e de testes de um modelo de sistema de recomendações de materiais didáticos, por meio de um regate teórico sucinto capaz de elucidar um método de recomendações adequado para o desenvolvimento de protótipo, e que possibilitasse além de regate teórico-prático. 0 estudo compreende parte das atividades desenvolvidos em curso de extensão realizado na Faculdade Estácio de Alagoas, no ano de 2010, junto à disciplina "Trabalho de Conclusão de Curso- TCC", oferecida pelo curso de Sistema de Informação da Faculdade Estácio de Alagoas.

O presente artigo está estruturado da seguinte forma: na seção 1 é abordado o resgate teórico referente aos sistemas de recomendação; a seção 2 trata sobre o resgate metodológico do modelo gerador de recomendações híbridas; a seção 3 aborda o desenvolvimento do protótipo utilizado para testar o modelo de sistema de recomendação; a seção 4 explica 
como foi realizado o teste do protótipo; a seção 5 mostra os resultados obtidos no teste; a seção 6 aborda as considerações finais obtidas com o presente estudo.

\section{Resgate teórico: sistema de reco- mendação}

De acordo com Burke (2002), um sistema de recomendação é uma ferramenta computacional capaz de gerar recomendações de itens com características de interesse dos usuários.

Um dos desafios do estudo de Sistemas de Recomendação está em selecionar alguns itens para serem recomendados aos usuários, em um conjunto variado de itens que pode variar entre centenas ou milhares de unidades, de modo a satisfazer as preferências de cada usuário (CAZELLA et al., 2010). Para ficar mais claro a dimensão desse problema, suponha-se que $\mathrm{C}$ seja o conjunto de usuários de uma determinada loja virtual, e que S seja o número de itens que essa loja disponibiliza para venda, que podem incluir livros, revistas, filmes, roupas, dentre outros. Então, o produto cartesiano de $\mathrm{C} \times \mathrm{S}$ pode ser um conjunto de combinações bem extenso. Um sistema de recomendação deve selecionar e mostrar para os usuários produtos que satisfaçam aos seus gostos, ou seja, produtos os quais estejam de acordo com seus perfis, reduzindo assim o overload cognitivo (MANOUSELIS; COSTOPOULOU, 2008).

Sistemas de recomendação buscam, também, otimizar a quantidade de produtos recomendada ao usuário. Para conseguir realizar essa tarefa, esses sistemas necessitam de informações sobre seus usuários e sobre os itens que serão recomendados, fator necessário para o cálculo do grau de utilidade de cada produto para um determinado usuário. O grau de utilidade $u$ de um item $c \in C$ indica o quanto esse item irá satisfazer, ou não, aos gostos de um determinado usuário $s \in \mathrm{S}$. Assim, um sistema de recomendação procura selecionar e recomendar a um determinado usuário apenas produtos que possuam os maiores graus de utilidade para este usuário. Adomavicius e Tuzhilin (2005) formalizaram esse problema como:

$$
\forall \mathrm{c} \in \mathrm{C}, \quad \mathrm{s}^{\prime}{ }_{\mathrm{c}}=\max _{\mathrm{s} \in \mathrm{S}} \mathrm{u}(\mathrm{c}, \mathrm{s})
$$

Em que, para todo usuário $c$, pertencente ao conjunto de usuários $C$, um sistema de recomendação tenha como objetivo recomendar apenas os produtos $\mathrm{s}^{\prime}$ o qual, para um determinado usuário $c$, maximize a função de utilidade $\mathrm{u}(\mathrm{c}, \mathrm{s})$, ou seja, possua um valor elevado de utilidade em relação aos demais produtos.

Schafer et al. (1999) definiram que os sistemas de recomendação podem ser classificados quanto a forma em que as recomendações são geradas, são eles: Recomendação não personalizada, Recomendação baseada nas características dos itens, Recomendação baseada na correlação entre os itens e Recomendação baseada na correlação entre as pessoas. No primeiro são consideradas as aquisições ou avaliações de um produto por um conjunto de usuários, de forma que um produto bem requisitado é indicado para outros usuários. O perfil do usuário não é levado em consideração, apenas a avaliação dos produtos. No o segundo leva em consideração o perfil do usuário, e o sistema deve possuir meios de efetuar a coleta de informações sobre as características dos usuários, que são usadas na hora de gerar as recomendações. O terceiro utiliza o histórico das aquisições do usuário, de modo que itens semelhantes aos já adquiridos no passado sejam também recomendados. Por fim, a Reco- 
mendação baseada na correlação entre pessoas analisa grupos de usuários com mesmas preferências, de modo que um item adquirido e bem avaliado por um indivíduo é recomendado para os membros do mesmo grupo.

Já Adomavicius e Tuzhilin (2005) definem que sistemas de recomendação podem ser baseados no conteúdo, baseados na filtragem colaborativa, ou híbridos.

Sistemas de recomendação baseados no conteúdo tentam premeditar o grau de utilidade de um determinado produto para um determinado usuário, com base em seu passado. A utilidade $\mathrm{u}(\mathrm{c}, \mathrm{s})$ de um produto $\mathrm{s}$ para $u m$ usuário c tem seu cálculo baseado na utilidade $\mathrm{u}\left(\mathrm{c}, \mathrm{s}_{\mathrm{i}}\right)$, tal que $\mathrm{s}_{\mathrm{i}}$ são os itens pertencentes ao conjunto $S$, adquiridos em algum momento do passado (ADOMAVICIUS; TUZHILIN, 2005). Essa técnica utiliza a análise do histórico da interação entre usuário e ambiente, com o objetivo de obter os produtos que foram adquiridos pelos usuários no passado (FELFERNIG; BURKE, 2008). Para serem feitas as recomendações dos itens, é necessária a existência de uma base de dados que contenha informações relativas às características dos produtos a serem recomendados. Além disso, o perfil do usuário também é necessário, já que essa técnica de recomendação baseia-se na realização do cruzamento das características do produto com o perfil do usuário, o qual é construído a partir da análise do histórico de suas aquisições anteriores (FELFERNIG et al., 2008). Essa abordagem, de geração de recomendações, possui uma limitação, de não poder gerar "surpresas" para o usuário, ou seja, não recomendar um produto que em um determinado momento esteja sendo bastante procurado por outros usuários. Essa limitação reduz as chances do sistema de recomendação identificar novas características do usuário, já que novi- dades não são oferecidas, mesmo que seja um item em "moda" entre os usuários.

Sistemas baseados na filtragem colaborativa tentam premeditar o interesse de uma pessoa em relação a novos itens, baseando-se nas recomendações feitas para outras pessoas com gostos similares (ANSARI; ESSEGAIER; KOHLI, 1999). É uma abordagem de sistemas de recomendação o qual, assim como Schafer et al. (1999) definiram, leva em consideração a correlação pessoa-pessoa. O problema pode ser formulado com uma matriz de usuários $x$ itens, onde cada célula da matriz armazena o grau de utilidade $\mathrm{u}(\mathrm{c}, \mathrm{s})$ do item $\mathrm{s}$ em relação a um usuário c. Esse poderá ser obtido pela avaliação do usuário em relação ao item recomendado. O trabalho está em premeditar essa utilidade para as células vazias, ou seja, premeditar a utilidade de um item para um usuário (MANOUSELIS; COSTOPOULOU, 2008), permitindo ao sistema de recomendação avaliar o grau de utilidade e, assim, decidir se o item será recomendado ou não. A técnica de Filtragem Colaborativa tenta premeditar a utilidade do item para o usuário, com base na utilidade do mesmo produto para um conjunto de usuários possuidores das mesmas características do usuário em questão. Uma das tarefas do sistema é identificar os grupos de usuários com características semelhantes, chamados de grupos de pares ou grupos de vizinhos, processo esse chamado de geração da vizinhança (SARWARET al., 2000). Esses grupos devem conter indivíduos com interesses ou comportamentos comuns (CAZZELA et al., 2010). 


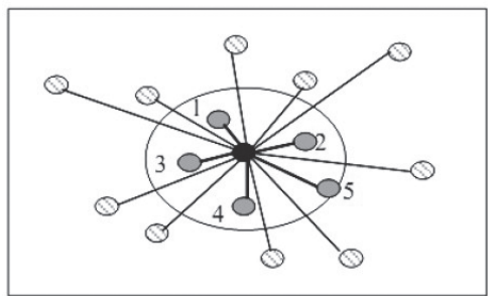

Figura 1: Formação de Vizinhança Fonte: Sarwar et al. (2000, p. 2).

Para Berkonsky et al. (2008), a Filtragem Colaborativa é uma das mais conhecidas técnicas para premeditar e gerar recomendações. Seu algoritmo consiste em montar uma matriz de pontuações, onde as linhas representam os usuários e as colunas os itens, de modo que sejam identificados grupos de usuários com as pontuações aproximadas. Nesse contexto, quanto mais próximas as pontuações, mais semelhantes são os perfis dos usuários. Já para Schafer et al. (2007), essa técnica de recomendação utiliza algoritmos conhecidos como user-base near est neighbor algorithms, pois utilizam como base os vizinhos mais próximos do usuário que receberá a recomendação.

Herlocker (2000) definiu que, para efetuar recomendações nessa abordagem, três passos devem ser seguidos:

1. Calcular o grau de similaridade de todos os usuários em relação à um determinado usuário, por meio da equação de Pearson:

$$
w_{a, u}=\frac{\sum_{i=1}^{m}\left[\left(r_{a, i}-\bar{r}_{a}\right)\left(r_{u, i}-\bar{r}_{u}\right)\right]}{\sqrt{\sum_{i=1}^{m}\left(r_{a, i}-\bar{r}_{a}\right)^{2} \sum_{i=1}^{m}\left(r_{u, i}-\bar{r}_{u}\right)^{2}}}
$$

A aplicação dessa equação indica o valor da correlação do usuário a em relação ao usuário $\mathrm{u}$, representado por $\mathrm{w}_{\mathrm{a}, \mathrm{u}}$. As variáveis $\mathrm{r}_{\mathrm{a}, \mathrm{i}} \mathrm{e}$ $\mathrm{r}_{\mathrm{u}, \mathrm{i}}$ indicam a avaliação do item i para o usuário a e $\mathrm{u}$, respectivamente; $\overline{\mathrm{r}}_{\mathrm{a}}$ e $\overline{\mathrm{r}}_{\mathrm{u}}$ indicam a média de todas as avaliações do usuário a e u, para os produtos por eles avaliados. Os resultados encontrados podem variar entre -1 e 1 .

Selecionar um subconjunto de usuários com similaridades de pesos mais altas. Ao aplicar a equação de Pearson aos usuários, chega a hora de identificar quais são os vizinhos mais próximos. É necessário estipular um $W_{\text {corte }}$, de forma que, quanto mais próximo esse valor for igual a 1, mais próximos serão os perfis dos usuários e, consequentemente, menor será o número de usuários selecionados. Entretanto, quanto mais próximo esse valor for igual a -1 , mais distantes serão os perfis dos usuários e, consequentemente, maior será o número de usuários selecionados. Assim, a escolha do valor de corte deve ser realizada de forma racional, para obtenção de um bom número de usuários vizinhos.

Normalizar as avaliações e computar a predição dos produtos para o usuário, por meio da equação:

$$
p_{a, i}=\bar{r}_{a}+\frac{\sum_{u=1}^{n}\left[\left(r_{u, i}-\bar{r}_{u}\right) * w_{a, u}\right]}{\sum_{u=1}^{n} w_{a, u}}
$$

A qual calcula a premeditação $\mathrm{p}_{\mathrm{a}, \mathrm{i}}$ do usuário a em relação ao produto i, levando em consideração o conjunto de usuários u que são vizinhos a a. Na equação, $\overline{\mathrm{r}}_{\mathrm{a}}$ e $\overline{\mathrm{r}}_{\mathrm{u}}$ indicam a média das avaliações do usuário a e u respectivamente, enquanto que $\mathrm{w}_{\mathrm{a}, \mathrm{u}}$ indica a similaridade do usuário u em relação ao usuário a. Para esse cálculo, somente os usuários com uma similaridade relativamente alta são considerados vizinhos e assim escolhidos para gerar as premeditações.

Um problema dos sistemas de recomendação baseados na filtragem colaborativa é tentar gerar recomendações sem a existência de muitas informações sobre os itens adquiridos no passado pelos usuários do sistema (BURKE, 
2002). Isso acontece normalmente com um novo usuário, tornando difícil para o sistema de recomendação compará-lo a outros usuários no processo de geração da vizinhança e, consequentemente, recomendar itens que satisfaça ao seu perfil.

Esse problema é conhecido como "Partida do novo usuário" (CAZELLA, 2006) ou Cold Start User (SCHAFER et al., 2007), e acontece quando o sistema de recomendação não possui informações suficientes sobre o usuário para gerar as recomendações.

Diante das limitações dos dois últimos tipos de sistemas apresentados, por meio das considerações Adomavicius e Tuzhilin (2005) percebeu-se que os sistemas de recomendação híbridos mais se aproximam das necessidades buscadas para a elaboração de um modelo de geração de recomendações para materiais didáticos, sendo assim este método será abordado com mais ênfase no tópico a seguir.

\subsection{Métodos de recomendação hí- bridos}

Um sistema de recomendação híbrido é definido como um sistema que combina duas ou mais técnicas de recomendação para poder indicar itens aos usuários (BURKE, 2002). Donaldson (2007) definiu que eles combinam a recomendação baseado no passado dos usuários com a recomendação baseada na colaboração dos usuários, ou seja, com base nas correlações item-item e na correlação pessoa-pessoa.

A vantagem de uma abordagem que unifique as outras é ampliar significativamente as chances de obter acertos em suas recomendações e eliminar as limitações de ambas abordagens. Os autores Burke (2002) e Adomavicius e Tuzhilin (2005) definiram alguns modos de como se obter tais sistemas. São eles:
- Implementando a recomendação com base no passado dos usuários e com base na colaboração dos usuários separadamente, combinando as pontuações das recomendações para gerar uma única recomendação;

- Realizando a escolha sobre o modo como a recomendação será feita, se baseada no conteúdo ou baseada na filtragem colaborativa. A escolha pode ser feita de forma aleatória, ou por critérios estipulados dentro do sistema.

- Gerando várias recomendações ao mesmo tempo, sendo que todas as técnicas de recomendação são utilizadas, o que permite ao usuário obter várias recomendações, geradas por diversas abordagens.

- Utilizando a geração de recomendação em cascata, em que uma técnica é usada para selecionar alguns itens, que são avaliados por outra técnica de recomendação.

- Construindo um modelo unificado que incorpore ambas as abordagens de recomendação.

A criação de um modelo de sistema de recomendação híbrido possibilita reduzir os problemas da partida fria e do novo usuário, já citado anteriormente, na tentativa de gerar recomendações mais próximas ao perfil do usuário.

\section{Resgate metodológico: modelo gerador de recomendações híbri- das}

A luz da revisão de literatura, que culminou na construção teórica sobre sistemas de recomendações do presente estudo, pertinente sinalizar o aporte metodológico elucidado por 
Ferro (2010) que por meio de testes discutiu e demonstrou a viabilidade do modelo de sistema de recomendações de materiais didáticos.

O olhar teórico de Ferro (2010), que pressupõe o caminhar metodológico, está baseado na colaboração de três modelos de recomendação, com base na natureza do contexto educacional ao qual o modelo foi concebido. São eles: recomendação não personalizada, recomendação baseada no conteúdo e recomendação baseada na filtragem colaborativa.

Desse modo, a primeira abordagem foi inserida no modelo por permitir que itens bem requisitados sejam recomendados, sem levar em consideração o perfil do usuário, para proporcionar que materiais didáticos de outras áreas de conhecimento também sejam sugeridos. Já a segunda abordagem foi inserida no modelo para proporcionar a recomendação de materiais didáticos com características semelhantes a outros já adquiridos no passado, ou seja, materiais de uma mesma área de conhecimento. Por fim, a recomendação baseada na filtragem colaborativa foi inserida para formação de grupos de estudantes com perfis semelhantes, dentro do ambiente virtual de aprendizagem, de modo a permitir que materiais didáticos adquiridos por algum membro do grupo também seja recomendado aos demais membros. Vejamos a figura 2 que ilustra o Modelo do Gerador de Recomendações Híbridas de Ferro (2010):

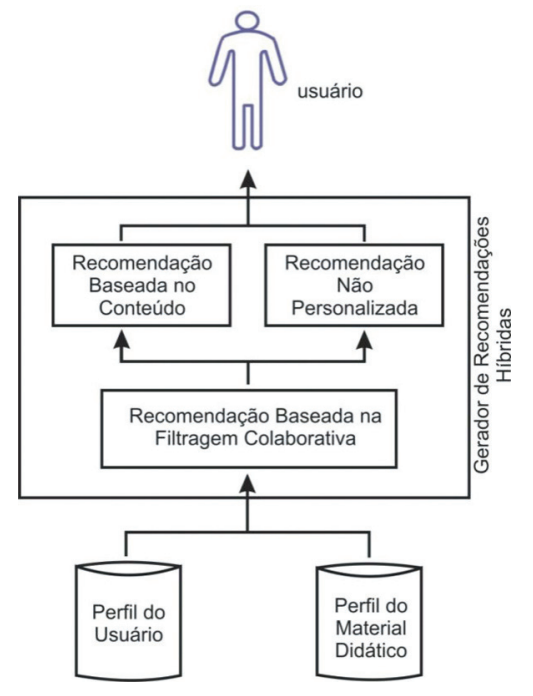

Figura 2. Modelo do Gerador de Recomendações Híbridas Fonte: Ferro (2010, p. 50).

O modelo consiste inicialmente em armazenar dados do usuário e de materiais didáticos. Os dados dos usuários armazenados são referentes às preferências de áreas de estudo, histórico de materiais já adquiridos no passado e avaliação das recomendações geradas.

O processo de geração de recomendações utiliza as seguintes etapas, de acordo com Ferro (2010):

1. É gerada uma lista de materiais didáticos, de itens ainda não adquiridos pelo usuário;

2. Os itens dessa lista recebem três valores, que são correspondentes aos valores de prioridades, ou ranking, calculado por meio das técnicas de recomendação:
a. Baseada na Filtragem colaborativa
b. Baseada no Conteúdo
c. Não Personalizada 
3. Para cada item é calculado o Grau de Utilidade Híbrida - uh(item), o qual consiste na equação:

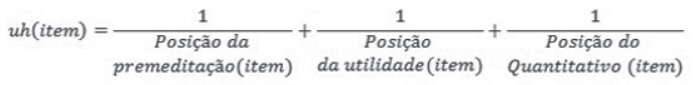

Figura 3. Fórmula do cálculo de utilidade híbrida Fonte: Ferro (2010, p. 51).

Entretanto, para a utilização das técnicas de recomendação citadas, foi necessário criar meios de extração de dados no AVA, para obtenção do Perfil do Usuário e Perfil do Material Didático. Assim, foi necessário criar alguns módulos, cada um com suas funcionalidades específicas.

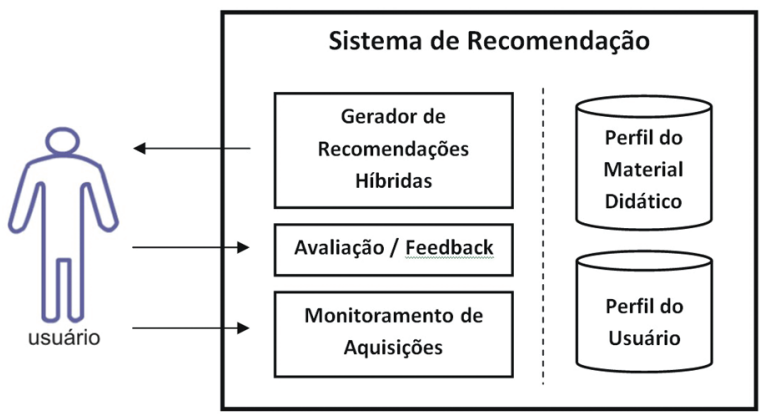

Figura 3. Arquitetura do Sistema de Recomendação Fonte: Ferro (2010, p. 46).

De acordo com a figura 3, a qual mostra a arquitetura do sistema de recomendação proposto, identificam-se:

- Gerador de Recomendações Híbridas- tem como papel efetuar o cruzamento dos dados do Perfil do Usuário com os dados do Perfil do Material Didático para gerar recomendações. Esse módulo utiliza o modelo definido na figura 2.

- Perfil do Usuário - dados referentes às características e interesses dos usuá- rios do AVA. A identificação do Perfil do Usuário foi realizada nas seguintes formas:

- Direta: por intermédio do preenchimento de um formulário pelo próprio usuário durante o seu cadastro.

- Indireta: pelo monitoramento das interações do usuário com o próprio sistema, que será realizado pelos componentes Avaliador/Feedback e Monitoramento de Aquisições.

- Perfil do Material Didático - características do material didático disponibilizado no AVA pelos professores. Essas características referem-se às áreas de conhecimento relacionadas ao conteúdo do material.

- Avaliação / Feedback - Trata-se de um componente pelo qual o usuário poderá avaliar as recomendações visualizadas, dando o seu feedback sobre a sugestão que lhe foi oferecida. A avaliação consistirá em informar se a recomendação gerada foi útil, ou seja, se o material didático sugerido possuía características similares às características do Perfil do Usuário. Se o usuário informar que a recomendação foi satisfatória, é sinal de que o material didático sugerido possui características que despertaram o seu interesse. Nesse caso, o Perfil do usuário é automaticamente atualizado, de modo a incrementar o peso da área de estudo correspondente ao material didático.

- Monitoramento de Aquisições - o usuário pode, dentro do AVA, obter material didático a qualquer momento, seja esse material composto com as características de interesse dele ou não. Assim, esse módulo tem por finalidade armazenar as informações de aquisição do usu- 
ário, sejam de materiais recomendados, não recomendados; sejam de materiais com características do seu perfil, ou características que não estavam em seu perfil.

- Gerador de Recomendações Híbridas - tem como papel efetuar o cruzamento dos dados do Perfil do Usuário com os dados do Perfil do Material Didático para gerar recomendações.

\section{Desenvolvimento de um protótipo}

Objetivando testar o modelo proposto, foi inicialmente realizado um levantamento de quais AVAs poderiam ser utilizados. Moodle, Teleduce e-ProInfo foram identificados como ambientes bastante utilizados no Brasil, no qual para efeito do presente trabalho o Moodle foi a ferramenta escolhida, por ser ambiente utilizado pela Universidade Federal de Alagoas em seus cursos de EAD, local onde foi iniciada essa pesquisa.

Portanto foi criado o sistema de recomendação, na forma de um módulo do Moodle, para realizar a recomendação assistida aos usuários. Em sua composição, foi utilizada a linguagem de programação PHP e banco de dados MySQL. Foi utilizada também a linguagem de estilo Cascading Style Sheets (CSS), proporcionando maior organização à codificação do layout das telas, e a linguagem de programação Java Script, para gerar a emissão de mensagens de saída para os usuários do ambiente.

Tornou-se necessário, também, desenvolver algumas modificações no Moodle, para permitir o funcionamento dos módulos definidos na arquitetura da Figura 3, segundo Ferro (2010). Foram criados:

- Perfil do Usuário - local onde os usuários podem informar, diretamente, as suas áreas de interesse. Foram os nomes de alguns cursos, e suas respectivas disciplinas, como forma de identificação das preferências de interesse dos usuários, conforme pode ser visualizado na figura 4.

- Perfil do Material Didático - local onde os usuários cadastrados com o perfil de professor no AVA podem efetuar o upload dos materiais didáticos, informando ao sistema as características referentes ao seu conteúdo, conforme mostra a tela do lado esquerdo da figura 4.

- Biblioteca de Materiais Didáticos - para centralizar e disponibilizar os arquivos com conteúdos acadêmicos, postados pelos professores, para alunos de quaisquer cursos. Essa área pode ser acessada a qualquer momento pelos usuários, além de permitir o download de qualquer material didático, mesmo que não possua as características do perfil do usuário; Ao solicitar o download de um material, o usuário é encaminhado para a área de Download do Material.

- Bloco de Recomendação Assistida - bloco do Moodle ao qual foi utilizado para mostrar as recomendações, além de permitir o acesso à biblioteca e à atualização do perfil do usuário, conforme pode ser observado no lado esquerdo da figura 5 . Ao clicar sobre a recomendação oferecida, o usuário é encaminhado para a área de Avaliação de Recomendação.

- Avaliação da Recomendação - área a qual o usuário onde o usuário informa se a recomendação fornecida foi boa, regular ou ruim, podendo ser visualizada no lado direito da figura 5. Ao avaliar a recomendação, o item ao qual foi sugerido pelo computador, o usuário faz 
a avaliação dessa sugestão. Ao avaliar a recomendação, o sistema encaminha o usuário para a área de Download do Material.

- Download do Material - Essa área, visualizada nas duas telas da figura 6,além de permitir o download do material didático, registra que o usuário adquiriu um item, armazenando em seu histórico de aquisições.

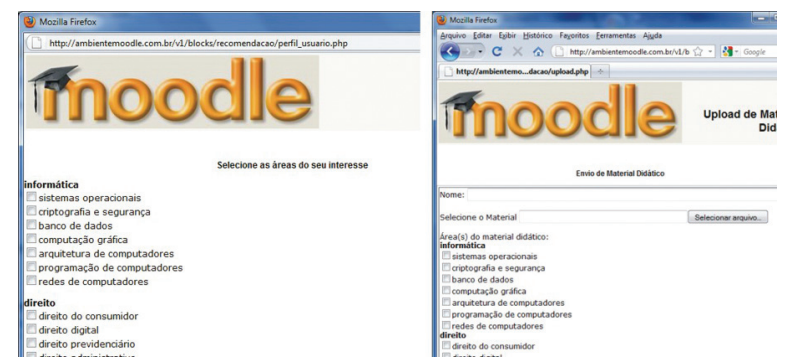

Figura 4. Área de atualização do Perfil do Usuário e local onde professores postam os materiais didáticos, informando o Perfil do Material Didático, respectivamente

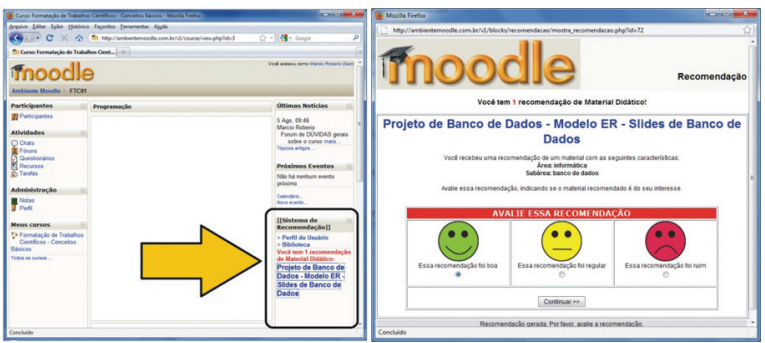

Figura 5. Recomendação Gerada e área onde o usuário avalia a recomendação, respectivamente

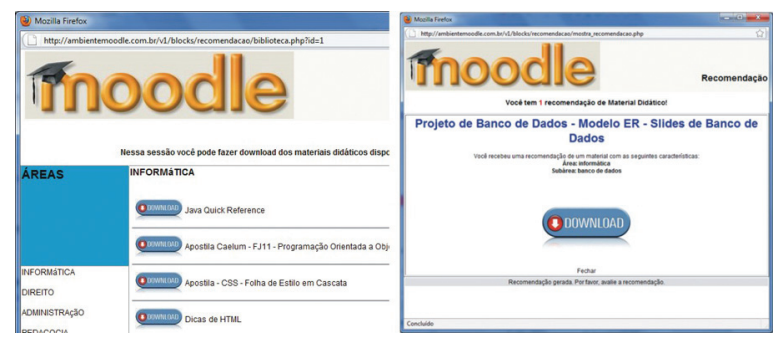

Figura 6. Biblioteca de Materiais Didáticos e Área de Download, respectivamente

\section{Testes}

O protótipo criado passou por alguns testes, que demonstraram funcionar como esperado. Os cálculos efetuados pelo Gerador de Recomendações foram conferidos e acompanhados, por meio de uma tela de acompanhamento de recomendações, exibida na Figura 7 , a qual mostra os valores referentes às técnicas de recomendação, de diversos materiais didáticos, para o usuário autenticado no sistema.

\begin{tabular}{|l|l|l|l|l||}
\hline $\begin{array}{l}\text { Material } \\
\text { Didático }\end{array}$ & $\begin{array}{l}\text { Utilidade Hibrida - } \\
\text { uh(material) }\end{array}$ & $\begin{array}{l}\text { Ranking do Predição - } \\
\mathrm{P}(\text { usuario,material) }\end{array}$ & $\begin{array}{l}\text { Ranking Grau de Utilidade } \\
\text { do Material Didático }\end{array}$ & $\begin{array}{l}\text { Não Personalizado - } \\
\text { downloads }\end{array}$ \\
\hline 1 & 0.7672413793103 & $2^{\circ}(\mathrm{P}=2.2938747552606)$ & $4^{\circ}$ (Peso $\left.=1\right)$ & $58^{\circ}(1$ downloads $)$ \\
\hline 2 & 1.58333333333333 & $1^{\circ}(\mathrm{P}=5.0293857574829)$ & $4^{\circ}$ (Peso $\left.=1\right)$ & $3^{\circ}(28$ downloads) \\
\hline 3 & 0.2984913793103 & $32^{\circ}(\mathrm{P}=0)$ & $4^{\circ}$ (Peso $\left.=1\right)$ & $58^{\circ}(1$ downloads $)$ \\
\hline 4 & 0.5505747126437 & $3^{\circ}(\mathrm{P}=2.2837338594102)$ & $5^{\circ}$ (Peso $\left.=0\right)$ & $58^{\circ}(1$ downloads $)$ \\
\hline 5 & 0.30125 & $32^{\circ}(\mathrm{P}=0)$ & $4^{\circ}$ (Peso $\left.=1\right)$ & $50^{\circ}(2$ downloads) \\
\hline \hline
\end{tabular}

Figura 7. Tela de Acompanhamento

Entretanto, para identificar se o modelo utilizado demonstrou gerar recomendações satisfatórias aos usuários, foi criado um curso de extensão na Faculdade Estácio de Alagoas, com o objetivo de analisar as recomendações sugeridas, além de verificar o feedback e interação dos usuários com o sistema em geral. O curso, intitulado Formatação de Trabalhos Científicos, promovido pelo professor da disciplina Trabalho de Conclusão de Curso, foi destinado aos alunos graduação e pós-graduação, e foi oferecido na modalidade à distância, com a carga horária de 20 horas, usando o ambiente Moodle e o protótipo do sistema de recomendação. A divulgação do curso foi feita em meio eletrônico, obtendo a quantidade de 71 alunos inscritos, de três faculdades distintas do Estado de Alagoas, distribuídos em diversos cursos de graduação. Participaram alunos da área de exatas, saúde e humanas, formando um grupo de alunos bem heterogêneo, com preferências e interesses de estudos distintos. 
Durante as atividades diárias do curso, textos e vídeos eram disponibilizados para os alunos, contendo embasamento para a realização de atividades propostas dentro do próprio ambiente. Recursos como o fórum e chat foram usados, proporcionando a interação entre aluno-professor. O conteúdo abordou assuntos relacionados a elaboração de pesquisas e de trabalhos científicos. Além disso, as aulas em vídeo tornaram o curso mais dinâmico, pois mostravam como formatar um trabalho, utilizando a ferramenta de edição de textos MS Word 2007 para criar sumários automáticos, numeração de páginas, notas de rodapé e geração automática da página de referências bibliográficas. As atividades iniciavam com um nível de complexidade baixo, aumentando gradativamente, até que, na última tarefa, foi solicitada a formatação de uma monografia disponibilizada para download.

$\mathrm{Na}$ biblioteca foram disponibilizados $71 \mathrm{ma}-$ teriais didáticos, de diversos assuntos e áreas de conhecimento. Esses materiais consistiam em artigos científicos, manuais, apresentações em Power Point e apostilas, todos em formato PDF. Antes de enviar os materiais para a biblioteca, foi necessário configurar as áreas e subáreas do sistema. Então, foram criadas 6 áreas: Sistemas de Informação, Direito, Administração, Pedagogia, Psicologia e Metodologia Científica. Quase todas possuíam nomes de cursos de graduação, com exceção da área chamada Metodologia Científica, incluída para disponibilizar manuais e textos sobre pesquisa, numa forma de complementar o conteúdo abordado em todo o curso. Cada área dividiase em outras subáreas, totalizando assim 28 subáreas, e cada material didático postado no ambiente tinha pelo menos uma subárea de conhecimento.

As mesmas áreas usadas para a inserção dos materiais didáticos era apresentadas para o usuário configurar o seu perfil. A configuração era solicitada no início do curso, por meio de um informativo, divulgando a necessidade dos alunos informar as suas preferências dentre as 28 subáreas listadas, iniciando a composição do Perfil do Usuário.

\section{Reflexões e resultados}

Dos 71 alunos inscritos, apenas 39 freqüentaram regularmente o ambiente, resultando numa evasão de aproximadamente $45 \%$. A maioria dos alunos desistentes alegou, também por email, a falta de tempo e a dificuldade em interagir com a AVA.

Ao total, foi constatado que foram geradas 207 recomendações de materiais didáticos aos usuários, resultando numa média um pouco superior a cinco recomendações por pessoa. Esse número reflete justamente a duração do curso, que foi de cinco dias. Entre todas as recomendações, 85 foram avaliadas pelos alunos, dado indicador de que nem todas as recomendações foram lidas, ou que nem todas as recomendações despertaram o interesse dos alunos para a sua avaliação.

Das 85 recomendações avaliadas pelos usuários, de acordo com a Tabela 1, verificou-se que $65,8 \%$ obtiveram o conceito Bom, número satisfatório para poucos dias de interação entre usuários e sistema de recomendação.

Tabela 1. Relação Conceito x Quantidade de Avaliações

\begin{tabular}{|l|c|c|}
\hline \multicolumn{1}{|c|}{ Conceito } & $\begin{array}{c}\text { Quantidade } \\
\text { de avaliações }\end{array}$ & $\begin{array}{c}\text { Porcenta- } \\
\text { gem }\end{array}$ \\
\hline Conceito Bom & 56 & $65,8 \%$ \\
\hline Conceito Regular & 21 & $24,7 \%$ \\
\hline Conceito Ruim & 8 & $9,5 \%$ \\
\hline Total & 85 & $100 \%$ \\
\hline
\end{tabular}


Em relação às demais avaliações, 24,7\% obtiveram conceito regular, indicando que a sugestão do material didático não foi boa, mas também não foi ruim. Sobraram, então, 9,5\% de avaliações negativas, valor pequeno, porém significativo, alvo de um estudo mais longo e aprofundado para conhecimento de suas causas.

\section{Considerações finais}

Diante dos estudos efetivados, o modelo de recomendação híbrido proposto apresentou-se satisfatoriamente nos testes iniciais e no experimento envolvendo alunos reais, em um curso também real. A arquitetura, em geral, permitiu ao sistema de recomendação absorver várias informações provenientes da interação dos usuários com o ambiente virtual de aprendizagem. Ao final do experimento, vários dados foram coletados, possibilitando a realização de várias análises e indicadores sugestivos para a realização de novas pesquisas.
De acordo com o levantamento realizado, 9,5\% das recomendações sugeridas tiveram avaliação negativa, sendo um dado motivador para a realização de novos experimentos e, consequentemente, melhorias no algoritmo gerador de recomendações.

Foi observada também a necessidade de novos experimentos com maior tempo de duração, permitindo ao sistema de recomendação evoluir e sugerir recomendações mais próximas do perfil do usuário. Como o modelo proposto utiliza a abordagem conhecida como Filtragem Colaborativa, a realização de experimentos com maior tempo pode contribuir para melhor entendimento do problema conhecido com Partida Fria e para a melhor geração de grupos de usuários com perfis semelhantes.

Por fim, as reflexões tecidas no presente artigo sinalizam para necessidade de novas pesquisas para o desenvolvimento de novas ferramentas capazes de possibilitar e favorecer o aprendizado e o acesso a informação em ambientes virtuais. Assim, espera-se que esse estudo seja visto como contribuição inicial para a discussão e início de novos estudos.

\section{Referências}

ABED, Relatório analítico da aprendizagem a distância no Brasil. Disponível em: <http://www. abed.org.br/censoead/censo2012.pdf>. Acesso em: 15 ago. 2013.

ADOMAVICIUS, G.; TUZHILIN, A. Toward the Next Generation of Recommender Systems: A Survey of the State-of-the-Art and Possible Extensions. IEEE Transactions on Knowledge and Data Engineering, p. 734-749, jun. 2005. Disponível em:

<http://www.computer.org/portal/web/csdl/doi/10.1109/TKDE.2005.99>. Acesso em: 10 jan. 2009.

ALMEIDA, M. E. B. D. Educação a distância na internet: abordagens e contribuições dos ambientes digitais de aprendizagem. Educação e Pesquisa, São Paulo, p. 327-340, 2003.

ANSARI, A.; ESSEGAIER, S.; KOHLI, R. Internet Recommendation Systems, Journal of Marketing 
Research. 1999. Disponível em: <http://www.atypon-link.com/AMA/doi/abs/10.1509/jmkr.37.3.363. 18779? cookieSet=1\&journalCode=jmkr $>$. Acesso em: 17 abr. 2010.

BERKONSKY, S.; EYTANI, Y.; MANEVITZ, L. Efficient Collaborative Filtering in Content-Addressable Spaces. Personalization Techniques and Recommender Systems, 2008. Cap 5, p. 135-164.

BRASIL. Portaria no 4059 de 10 de dezembro de 2004. Disponível em <http://portal.mec.gov.br/ sesu/arquivos/pdf/nova/acs_portaria4059.pdf>. Acesso em: 20 de ago. 2013.

BURKE, R. Hybrid Recommender Systems: Survey and Experiments. User Modeling and UserAdapted Interaction, v. 12, p. 331-370, 2002. Disponível em: <http://portal.acm.org/citation. cfm?id=586352>. Acesso em: 10 jan. 2009.

CAZELLA, S. C. Aplicando a Relevância da Opinião de Usuários em Sistemas de Recomendação para Pesquisadores. 2006. 180f. Tese (Programa de Pós-Graduação em Computação) - Instituto de Informática, Universidade Federal do Rio Grande do Sul, Porto Alegre.

CAZELLA, S. C.; REATEGUI, E. B. Sistemas de Recomendação. XXV Congresso da Sociedade Brasileira da Computação. 2006, São Leopoldo, p. 306-348.

CAZELlA, S. C.; NUNES, M. A. S. N.; REATEguI, E. B. A Ciência da Opinião: Estado da arte em Sistemas de Recomendação. XXX Congresso da SBC, 2010, Belo Horizonte.

DONALDSON, J. A Hybrid Social-Acoustic Recommendation System for Popular Music. ACM Conference On Recommender Systems, p. 187-190, 2007. Disponível em: <http://portal.acm.org/citation.cfm?id=1297271>. Acesso em: 07 jun. 2010.

FELFERNIG, A.; BURKE, R. D. Constraint-based recommender systems: technologies and research issues. ACM International Conference Proceeding Series. Vol. 342, 2008. Disponível em: <http:// portal.acm.org/citation.cfm? id=1409544>. Acesso em: 10 jan. 2009.

FELFERNIG, A.; TEPPAN, E.; GULA, B. User Acceptance of Knowledge-based Recommenders. Personalization Techniques and Recommender Systems, 2008. Cap 10, p. 249-275.

FERRO, M. R. C. Um Modelo de Sistema de Recomendação de Materiais Didáticos para Ambientes Virtuais de Aprendizagem. 2010. 97f. Dissertação (Programa de Mestrado em Modelagem Computacional de Conhecimento) - Instituto de Computação, Universidade Federal de Alagoas. Maceió.

Figueiredo, L. C. R; ALMeidA, D. J. R. A Tutoria Presencial na EAD. 2010. Disponível em: < http:// www.abed.org.br/congresso2010/cd/152010190142.pdf>. Acesso em: 26 fev. 2011. 
HERLOCKER, J. L. Understanding and Improving Automated Collaborative Filtering Systems. 2000. 144p. Tese. University of Minnesota, Minnesota, EUA.

MAIA, M. C. O uso da tecnologia de informação para a Educação a Distância no Ensino Superior. 2003. Tese (Doutorado em Administração de Empresas) - Escola de Administração de Empresas de São Paulo da Fundação Getulio Vargas, São Paulo.

MANOUSELIS, N.; COSTOPOULOU, C. Experimental Analysis of Multiattribute Utility Collaborative Filtering on a Synthetic Data Set. Personalization Techniques and Recommender Systems, 2008, Cap 5, p. 111-133.

SARWAR, B.; KARYPIS, G.; KONSTAN, J.; RIEDL, J. Analysis of Recommendation Algorithms for E-Commerce. Electronic Commerce. p. 158-167, 2000. Disponível em: <http://portal.acm.org/citation. cfm?id=352887>. Acesso em: 10 jan. 2009.

SCHAFER, J. B.; FRANKOWSKI, D.; SEN, S; HERLOCKER, J. Collaborative Filtering Recommender Systems. The Adaptive Web. V. 4321/2007, p. 291-324, 2007. Disponível em: <http://www.springerlink. com/content/t87386742n752843/ export-citation/>. Acesso em: 11 jun. 2009.

SCHAFER, J. B.; KONSTAN, J.; RIEDL, J. Recommender Systems in E-Commerce. Electronic Commerce: Proceedings of the 1st ACM conference on Electronic commerce. p.158-166, 1999. Disponível em: <http://portal.acm.org/citation.cfm ?id=337035>. Acesso em: 11 jan. 2009.

Submetido para avaliação em 03 de junho de 2013.

Aprovado para publicação em 26 de outubro de 2013.

Márcio Robério da Costa Ferro: Analista de Tecnologia da Informação do Instituto Federal de Educação, Ciências e Tecnologia de Alagoas - Maceió - AL - Brasil. E-mail: mrcferro@gmail.com

Fábio Paraguaçu Duarte da Costa: Professor adjunto na Universidade Federal de Alagoas - Maceió - AL - Brasil. E-mail: fabioparagua2000@gmail.com

Angela Lima Peres: Professora Titular Fundação Universitária de Ciências da Saúde de Alagoas - Maceió - AL Brasil. E-mail: limaperes@gmail.com

Marcela Ferreira Marinho: Professora da Faculdade Estácio de Alagoas - Maceió - AL - Brasil. E-mail: marcelaferreiramarinho@gmail.com 